\title{
Una intervención formativa del Centro de Escritura Nebrija en la Facultad de Cien- cias Sociales
}

\author{
A formative intervention of Nebrija Writing Center at the Faculty of Social Sciences
}

\author{
Alicia de la Peña Portero (iD \\ e-mail: apena@nebrija.es \\ Universidad Nebrija. España
}

\author{
Rocío Santamaría Martínez \\ e-mail: rsantama@nebrija.es \\ Universidad Nebrija. España
}

\section{Resumen}

La enseñanza y adquisición de la competencia escrita es trascendental para el avance de la sociedad del siglo XXI. Por ello, se hace necesario desarrollar la alfabetización académica de los estudiantes y futuros profesionales en las universidades, incluyendo en los planes de estudio de cada facultad la escritura académica integrada en las disciplinas. Asimismo, es fundamental crear centros de escritura y facilitar la formación continua de los escritores a través de talleres específicos, tanto presenciales como en línea. Este trabajo ilustra una exitosa experiencia transversal conjunta entre el Centro de Escritura y la Facultad de Ciencias Sociales de la Universidad Nebrija. El objetivo de la intervención formativa del centro de escritura en la asignatura de Diseños de investigación en Psicología es facilitar a los estudiantes las técnicas y las estrategias necesarias para la redacción de un artículo científico. Se presentan el método y las fases de trabajo y se analizan los resultados obtenidos. Para esta investigación descriptiva se ha aplicado un proceso de observación cualitativa a través de entrevistas con el docente, cuestionarios de percepción, rúbricas de revisión y encuestas de calidad de escritores y seguimiento de los tutores; todo ello ha hecho medible la experiencia de intervención. Como conclusión, debería contemplarse en todos los programas de grado la implementación de planes de escritura en las distintas disciplinas con el fin de que los estudiantes desarrollen hábitos escritores y aumenten su adecuación en los distintos géneros discursivos.

Palabras clave: centro de escritura; alfabetización académica; escritura en las disciplinas; psicología; competencia comunicativa.

\begin{abstract}
The teaching and acquisition of the writing competence is key for the progress of the 21 st century society. Therefore, it is necessary to foster students and future professionals' academic literacy in university, integrating academic writing in the curricula of the different disciplines in each department. Additionally, it is essential to create writing centers and promote writers' continuous education with specific workshops, both in class and online. This paper depicts a successful transversal experience shared by Nebrija University Writing Center and the Social Sciences Department. The objective of the writing center's formative intervention in the subject Research Designs in Psychology is to provide students with the necessary techniques and strategies to write a scientific article. The methodology and work phases are presented and the results analysed. For this descriptive research a qualitative observation process is applied by means of interviews with the professor, perception questionnaires and revision rubrics, as well as quality surveys and tutors follow up. All of these make the intervention experience measurable. As a conclusion, every degree program should consider implementing writing plans in the different disciplines so that students develop writing habits and increase their adequacy in the different discourse genres.

Keywords: writing center; academic literacy; writing in the disciplines; psychology; communicative competence.

Recibido / Received: 26-01-2020

Aceptado / Accepted: 21-04-2020

Publicado en linea / Published online: 01-07-2020
\end{abstract}

Cómo referenciar este artículo / How to reference this article:

de la Peña Portero, A. \& Santamaría Martínez, R. (2020). Una intervención formativa del Centro de Escritura Nebrija en la Facultad de Ciencias Sociales. Tendencias Pedagógicas, 36, pp. 91-103. doi: 10.15366/tp2020.36.07 


\section{Introducción}

En un mundo interconectado y global, la comunicación interpersonal y el intercambio de información han cobrado tal relevancia que hoy día se puede hablar de comunicación mediada por ordenador (Herring, 1984; Kern, 2006). Esta comunicación, con la llegada y la integración de las nuevas tecnologías en la práctica totalidad de las esferas de la vida, se realiza, en su mayor parte, de forma escrita (Puerta \& Sánchez, 2010).

Teniendo esto en cuenta, es necesario reivindicar la complejidad que supone la elaboración de textos escritos y la cantidad de operaciones cognitivas que conlleva su proceso de elaboración. Por esta razón, el desarrollo de la competencia comunicativa escrita es de especial transcendencia en todos los niveles educativos, especialmente en la universidad, ya que es necesario familiarizar a los estudiantes con los textos y el lenguaje propios de diversas comunidades discursivas, tanto académicas como profesionales. De igual modo, es fundamental facilitarles el acceso y la integración a aquellas comunidades profesionales de su interés poniendo en evidencia, al mismo tiempo, la importancia de un aprendizaje a lo largo de toda la vida.

La dificultad que entraña el desarrollo de la competencia comunicativa escrita se hace evidente en este nivel universitario dado que, en muchas ocasiones, los estudiantes llegan con poca experiencia escritora y se enfrentan por primera vez a culturas discursivas nuevas (Carlino, 2005), cada una con sus propios códigos y especificaciones. «La queja más generalizada de los docentes en la mayoría de países hispanohablantes es que los estudiantes universitarios no saben escribir (Gutiérrez-Rodríguez \& Flórez-Romero, 2011) y que tienen dificultades para leer y producir textos académicos (ÁvilaReyes, 2017; García Negroni \& Hall, 2010; Núñez Cortés, 2013).» (de la Peña, 2019, p. 75).

En líneas generales, los estudiantes universitarios presentan problemas a la hora de seleccionar, leer, analizar fuentes documentales y, por supuesto, de expresar y argumentar sus opiniones, construir una macroestructura textual correcta y adecuada, elaborar textos coherentes y cohesionados, identificar diferentes registros y producir los diferentes modos de organización del discurso. Tampoco parecen plenamente conscientes de las diferentes fases de las que consta el proceso de elaboración de textos escritos ni dominan completamente los procedimientos discursivos propios de los documentos académicos como la selección y citación bibliográfica o la reformulación de ideas. Todas estas dificultades se acompañan de una competencia lingüística, en ocasiones, insuficiente (pobreza léxica, problemas de ortografía, puntuación y acentuación, etc.) (Álvarez, Villardón, \& Yániz, 2010; Arrieta et al., 2006; Avilán, 2004; Bartolomé 2004; Carlino, 2005; Gallego \& Mendías, 2012; Lea \& Street, 1998; Ochoa \& Aragón, 2007; Reguera, 2010; Sabaj, 2009; Tapia, Burdiles, \& Arancibia, 2003; Vázquez, 2005).

Por todo ello, resulta de vital importancia desarrollar la comunicación escrita en el ámbito universitario y, en consecuencia, la alfabetización académica, como principal guía para el dominio de las diferentes culturas escritas. Son múltiples las iniciativas que se han puesto en marcha en el marco de la alfabetización académica en el ámbito de la educación superior. De entre ellas, se destacan en este artículo las acciones del movimiento Writing Across the Curriculum (WAC), los centros de escritura y los talleres de escritura para estudiantes.

En este contexto se enmarca la intervención formativa que aquí se detalla, en la que el Centro de Escritura Nebrija (CEN) colabora con el docente de la asignatura Diseños de investigación en Psicología para la formación de estudiantes del grado en Psicología en la escritura colaborativa de artículos científicos. En los siguientes apartados se presenta, en primer lugar, el marco teórico sobre el que se sustenta esta experiencia. A continuación, se describen, tanto el contexto y la muestra del estudio, como el diseño de la intervención formativa que los tutores del CEN llevan a cabo en el aula. Se termina con la presentación de los resultados más relevantes, así como de las conclusiones que se derivan de esta intervención colaborativa.

\section{Marco teórico}

En este apartado se resumen los principales pilares teóricos sobre los que se asienta este estudio. En primer lugar, se expone la concepción de la escritura que guía esta experiencia, entendiéndola como un proceso complejo y una herramienta imprescindible para la construcción del conocimiento. Asimismo, se hace hincapié en la importancia de la alfabetización académica en la universidad y se explican brevemente algunas de las iniciativas que en ella se enmarcan y que se han incorporado a esta 
intervención formativa, como las del movimiento Writing Across the Curriculum (WAC), los centros de escritura, el CEN, en particular, y los talleres de escritura.

\subsection{La escritura como proceso}

Un texto es un instrumento necesario para la construcción del pensamiento y, por tanto, su elaboración ha de entenderse como un proceso epistémico complejo en el que han de ponerse en práctica diversas habilidades cognitivas. Actualmente, además, la mayoría de los expertos abordan la escritura desde una perspectiva sociocultural en la que no solo se tienen en consideración dichos procesos y operaciones cognitivos, sino que también se valora la importancia del contexto en el que un texto se escribe y del que depende su elaboración (Arnáez, 2008; Castelló, Bañales, \& Vega, 2010; De Beaugrande \& Dressler, 1997; Milian, 2001; Cassany \& Morales, 2008; Wertsch, 1991).

Para comprender esta complejidad, es necesario remontarse al modelo propuesto por Flower y Hayes (1980) en el que se señala que durante la elaboración de un texto se distinguen tres fases principales. En primer lugar, la planificación, momento en el que se elige el objetivo del tema, se determinan las ideas principales y se organiza la información, siempre teniendo en cuenta el contexto discursivo en el que se inserta el texto. Le sigue la fase de textualización, que se corresponde con el acto de redacción en sí mismo. Por último, el proceso de revisión, durante el que se relee y se reformula la producción escrita. Estas etapas no son independientes, sino que se suceden de forma circular durante el ejercicio de producción textual y, por tanto, son concurrentes (se pueden dar dos o más etapas al mismo tiempo), iterativas (todas las fases son susceptibles de ser repetidas) y recurrentes (no es necesario terminar una operación para que empiece la siguiente) (Caldera \& Bermúdez, 2007; de la Peña, 2019).

Dicho marco procesual ha dado lugar a numerosas investigaciones posteriores de entre las que destacan los trabajos de Bereiter y Scardamalia (1987) y Thornbury (2006), que distinguen dos modelos de escribir. Por un lado, el denominado «decir el conocimiento» (product writing), en el que el objetivo principal es reproducir información de forma más o menos literal. Por otro lado, el llamado «transformar el conocimiento» (process writing), según el cual «writers do not in fact start with a clear idea of the finished product. Rather, the text emerges out of a creative process». (Thornbury, 2006, p. 249). Así, el texto ya no puede considerarse solo un producto final, sino que su elaboración abarca todo este proceso en el que lo deseable es que el escritor transforme el conocimiento y se apropie de él generando conocimiento nuevo respetando las necesidades retóricas exigidas por cada tipo de texto.

Este modelo de escritura entendida como proceso para el desarrollo de la competencia escrita es el que tratan de implementar la mayoría de los centros de escritura universitarios, tanto en España como en otros países, a través de sus tutorías entre pares (en las que estudiantes con una adquirida alfabetización académica ayudan a otros compañeros en las distintas fases del proceso de la escritura, según sus necesidades) y de diferentes intervenciones formativas en colaboración con docentes de distintas especialidades de grado y posgrado.

\subsection{La alfabetización académica en la enseñanza superior}

Resulta clara la relevancia de la alfabetización académica en la universidad como proceso de enseñanza que favorece «el acceso de los estudiantes a las diferentes culturas escritas de las disciplinas» (Carlino, 2013, p. 370). Esta importancia se revela aún mayor cuando se tienen en cuenta sus dos objetivos principales. Por un lado, «formar [a los estudiantes] para escribir y leer como lo hacen los especialistas» (Carlino, 2013, p. 370). Es decir, los universitarios deben aprender a comunicarse como los miembros de la comunidad universitaria de la que son parte al igual que como los miembros de las comunidades discursivas profesionales de las que pretenden formar parte en su futuro (Carlino, 2013). Asimismo, han de ser conscientes de su propio proceso de escritura, de los recursos de los que disponen y de los diferentes géneros y modos de escribir que les serán necesarios (Camps, 2011; Carlino, 2003; Lacon y Ortega, 2008; Marín, López, \& Roca-De-Larios, 2015). El segundo objetivo de la alfabetización académica es ayudar a los estudiantes a apropiarse del conocimiento producido por ellos durante el proceso de escritura. Esto es posible gracias al alto potencial epistémico que alberga la escritura como herramienta para desarrollar los procesos cognitivos implicados en el aprendizaje (Britton, 1970; Carlino, 2003; Cartolari \& Carlino, 2012; Lavellee, 2003; Marinkovich, Morán, 
\& Vergara, 1996). En resumen, «escribir también es una forma de aprenden» (de la Barrera \& Bono, 2004, p. 33).

La creciente atención que ha suscitado la alfabetización académica ha dado lugar a múltiples iniciativas que pretenden desarrollar la competencia comunicativa escrita en la universidad. De especial interés para este artículo son las que lleva a cabo el movimiento Writing Across the Curriculum (WAC), la creación de centros de escritura y los diferentes talleres y seminarios de escritura que se implementan en diferentes momentos del curso.

\subsubsection{Algunas iniciativas del movimiento Writing Across the Curriculum (WAC)}

El movimiento Writing Across the Curriculum (WAC) parte de la premisa de que los estudiantes solo asimilan lo que se les enseña si se involucran de forma activa en los temas de cada materia; por tanto, escribir sobre temas específicos de su disciplina de interés es una forma de apropiarse de esos contenidos. En líneas generales, esta tendencia es partidaria de unas prácticas docentes que conjuguen el saber sobre la materia en cuestión con el modo en el que la comunidad discursiva correspondiente trasmite, discute y comparte dicho conocimiento. Esto favorece el dominio de los diferentes géneros y modos discursivos, mejora la calidad de los textos y aumenta el conocimiento científico (Álvarez Angulo, 2011; de la Peña, 2019; Marín, 2006; Rojas \& Jiménez, 2012).

El movimiento WAC tiene una larga y asentada tradición en Inglaterra y Estados Unidos, donde se suele capacitar y apoyar a los docentes para que integren la escritura en sus cursos; sin embargo, en la mayor parte de la Unión Europea, el tratamiento de la escritura académica en el sistema universitario observa todavía cierta heterogeneidad que dificulta el establecimiento de un conocimiento compartido sólido. En el caso de España en particular, aunque es probable que también existan iniciativas WAC, todavía no parecen ser una práctica de trabajo habitual en la enseñanza superior. Es seguro que se estarán realizando proyectos aislados en esta línea, ayudando a los autores a escribir de manera efectiva y a desarrollar un pensamiento crítico y favoreciendo la alfabetización académica de la comunidad universitaria, pero, al mantenerse dentro del aula, hasta el momento apenas han recibido difusión científica.

En este sentido, en el caso particular del CEN, destacan las intervenciones formativas que está coordinando con algunos docentes de distintos grados de la universidad para incorporar la escritura en sus programas académicos dentro de las aulas, con el fin de que los estudiantes integren la alfabetización lectoescritora en su ámbito concreto de estudio y de manera transversal. Este es el contexto en el que se enmarca la experiencia que se llevó a cabo en el grado de Psicología en el segundo semestre del curso 2018-2019 y que se desarrolla en este artículo.

\subsubsection{Los centros de escritura en las universidades}

Otra de las iniciativas de la alfabetización académica es la creación de centros de escritura. Estos centros son un servicio que las universidades $-\mathrm{O}$, en ocasiones, una facultad o instituto universitario de lenguas - ofrecen a los estudiantes y a otros miembros de la comunidad universitaria con el objetivo de asesorarlos y guiarlos en la mejora de las prácticas escritas relacionadas con su desarrollo universitario y profesional.

Los centros de escritura se cimentan sobre la base del constructivismo social, en particular, la teoría sociocultural de la mente (Vigotski, 1978), de acuerdo con la cual la interacción es la fuente misma del aprendizaje. Por ello, los centros de escritura cuentan con tutores pares, normalmente estudiantes de posgrado o de los últimos años de grado que, tras una selección y formación específica impartida por los propios centros, actúan como lectores intermedios y asesoran a los tutorandos, previa cita, en cualquier momento del proceso de escritura. Esta interacción necesaria, a su vez, ayuda a poner en práctica una serie de competencias transversales altamente valoradas, tanto en el ámbito académico como en el profesional, y que todo estudiante universitario debería adquirir y perfeccionar. como son el trabajo en equipo, la autocrítica, la responsabilidad, la negociación o la toma de decisiones (Gibbs 
\& Simpson, 2004; Gómez Devís, 2015; Gómez, García, \& Saneleuterio, 2017; Ibarra \& Rodríguez, 2010).

Asimismo, estos centros diseñan y facilitan a la comunidad escritora recursos de escritura (esquemas textuales, consejos para escribir correctamente, rúbricas de corrección, técnicas de búsqueda de documentación, etc.) con carácter divulgativo.

A pesar de que no es un tema central de este artículo, conviene recordar que la tradición de los centros de escritura en las universidades está muy consolidada en el mundo anglosajón (Estados Unidos e Inglaterra) y en varios países de América Latina (Colombia, Chile, Argentina o México), y que sus largas trayectorias de investigación e implementación de la alfabetización académica han reportado, por ello, resultados muy positivos (Núñez Cortes, 2013). En comparación, en el caso de España, donde la alfabetización académica aún no tiene esa trayectoria tan consolidada, hoy solo se encuentran centros de escritura en ocho universidades, aunque con diferente grado de desarrollo y alcance.

\subsubsection{Los talleres de escritura en las universidades}

La última iniciativa de alfabetización académica que se quiere mencionar en este artículo son los talleres, cursos o seminarios sobre escritura académica que se ofrecen en las universidades en diferentes momentos de la etapa universitaria. En la mayoría de los casos se realizan al inicio de la etapa universitaria como herramienta remedial con el objetivo de homogeneizar el nivel de competencia comunicativa escrita de los estudiantes de nuevo ingreso a la vez que se les prepara para que se desenvuelvan con éxito durante sus años universitarios (Carlino, 2005; Núñez Cortés, 2013) y puedan realizar con éxito los trabajos finales de grado y posgrado.

Aunque estos talleres, a priori, pueden parecer una buena idea, suelen estar desconectados de las asignaturas de las diferentes titulaciones y a los estudiantes les resulta bastante difícil aplicar lo que aprenden en ellos al resto de sus materias. Además, con frecuencia estos cursos se centran en la corrección formal desatendiendo aspectos como la coherencia y la cohesión, el contexto de escritura y las convenciones discursivas de las diferentes especialidades. Por último, su mera existencia hace que la comunidad universitaria considere erróneamente que la alfabetización académica ya está cubierta y no es necesario seguir contribuyendo a su desarrollo, lo que hace que nadie se sienta responsable de esta parte de la formación del alumnado universitario.

Afortunadamente, poco a poco se está produciendo un cambio de mentalidad respecto de estos cursos y empiezan a integrarse en las propias asignaturas. Así, especialistas en Lengua, Análisis del Discurso, Educación o alfabetización académica están colaborando con expertos de diferentes disciplinas en la coordinación de programas y secuencias de trabajo de escritura específica (Carlino, 2013; Solé, Teberosky, \& Castelló, 2012).

\section{La descripción de la intervención}

En este apartado se presenta una intervención formativa concreta y conjunta entre el CEN y el docente encargado de la asignatura Diseños de investigación en Psicología del grado de Psicología de la Universidad Nebrija. En ella se ponen en práctica diferentes iniciativas de alfabetización académica del movimiento WAC, como un taller sobre los artículos científicos diseñado a medida para la experiencia y el trabajo colaborativo entre los estudiantes de Psicología y los tutores del CEN. Se describen, a continuación, el contexto de intervención, la muestra y el diseño de esta.

\subsection{El contexto de la intervención}

La intervención formativa que aquí se describe tiene lugar en la Facultad de Ciencias Sociales de la Universidad Nebrija (Madrid), en el marco de la asignatura Diseños de investigación en Psicología, que se imparte durante el segundo semestre del segundo año del grado en Psicología. Los objetivos principales de esta asignatura son, por un lado, dotar a los alumnos de los conocimientos suficientes para poder estudiar los procesos y fenómenos probabilísticos asociados a los factores de decisión humana, a las patologías clínicas y a las etapas sociales de pensamiento en todos los entornos de incertidumbre en los que se desarrolla la actividad profesional de la Psicología. Asimismo, se aspira a 
que los estudiantes desarrollen su competencia comunicativa escrita para transmitir información, ideas, problemas y soluciones a un público con niveles de especialización diversos.

Respecto del CEN, cabe señalar que es un servicio gratuito que nació con el curso 2018-2019 y que, desde el Instituto Nebrija para el Desarrollo de Competencias, se ofrece a toda la comunidad universitaria (a más de 3500 estudiantes en tres campus) con el fin de fomentar el desarrollo de la alfabetización académica. El CEN tiene como misión orientar y ayudar a los estudiantes Nebrija a desarrollar hábitos saludables y productivos de escritura académica y profesional, tanto en español como en inglés. Este servicio facilita formación específica (mediante talleres) y acompañamiento (a través de tutorías entre pares realizadas por sus más de 20 tutores, estudiantes voluntarios nacionales e internacionales, en su mayoría de últimos años de grado o masterandos) sobre los distintos tipos de textos universitarios más frecuentes (reseñas, resúmenes, ensayos, artículos científicos, trabajos de investigación, memorias de prácticas, Power Point, etc.). También apoya a los estudiantes que tienen dudas de carácter ortográfico, léxico y gramatical, asiste con la búsqueda de fuentes documentales y esclarece detalles relacionados con las normas de citación y referencias. Para atender esta demanda, el CEN se sirve del correo electrónico, de sus publicaciones en el campus virtual Nebrija y de las frecuentes publicaciones que se realizan en las redes sociales de la universidad, especialmente en la propia cuenta de Twitter del Centro (@de_escritura).

Dentro de su plan de acción tutorial de la escritura, el CEN también apoya a los profesores de las diferentes facultades que desean incluir la escritura en sus disciplinas, facilitándoles modelos textuales, actividades de escritura y rúbricas. Además, ofrece talleres formativos dentro del programa de la asignatura sobre distintos tipos de textos y realiza intervenciones formativas con sus tutores en clase. Durante estas intervenciones en el aula, un grupo de escritores y un tutor planifican el texto, los estudiantes lo van construyendo, desarrollan estrategias de lectura y escritura y los tutores, como lectores intermedios, dialogan con ellos y les guían. En esta interacción se promueve el componente social y dialógico (es decir, colaborativo) de la escritura y se desarrolla el componente afectivo, óptimo en todos los procesos escritores.

\subsection{La muestra de la intervención}

La muestra de la presente intervención está conformada por los estudiantes matriculados en los dos grupos de la asignatura Diseños de investigación en Psicología, que se imparte en el segundo semestre del curso 2018/2019 en la citada universidad. El total de estudiantes asciende a 55, 42 mujeres y 13 hombres de entre 18 y 34 años. Puesto que la Universidad Nebrija tiene una larga trayectoria de internacionalización, integra en sus aulas estudiantes de otras nacionalidades que vienen a España a estudiar, bien un semestre, bien un año completo de sus grados. Así, del total de 55, en este estudio 
se incluyen 15 estudiantes extranjeros de Venezuela (4), México (4), Argentina (2), Colombia (2) República Dominicana (1), Italia (1) y Holanda (1). Para este trabajo, los estudiantes se organizan en un total de 12 grupos.

Respecto de la participación del CEN, ocho tutores de español del curso 2018/2019 llevan a cabo esta formación colaborativa en el aula. Dichos tutores son, como ya se ha señalado, estudiantes voluntarios de último curso de grado o de posgrado.

\subsection{El diseño de la intervención}

Los objetivos que el CEN, a través de sus tutores, se propone con esta intervención formativa son:

- Ayudar a los estudiantes de Psicología a poner en práctica, implementar y comunicar los conocimientos adquiridos en el desarrollo de la asignatura Diseños de investigación en Psicología mediante la redacción de un artículo científico, convirtiendo esta práctica en una experiencia social y académica.

- Apoyar a los escritores para que mejoren la calidad de la escritura de sus artículos de investigación.

- Analizar de forma descriptiva las puntuaciones obtenidas por los alumnos de esta intervención del CEN, además de conocer su percepción y valoración del Taller de Escritura Académica de Supervivencia (TEAS). Esto permitirá mejorar la calidad y efectividad de las actividades del Taller para futuras ediciones.

La intervención formativa que se describe en este artículo consta de tres sesiones de aula en las que los tutores del CEN colaboran con los estudiantes de grado implicados en la elaboración de los textos requeridos por su profesor. Se procede, a continuación, a describir en detalle cada una de esas sesiones, además de las actividades que los estudiantes llevaron a cabo entre ellas.

Durante la primera sesión, miembros del CEN imparten un taller formativo presencial de redacción titulado «Cómo escribir un artículo científico». En él se explica cuál es el objetivo principal, el formato, la estructura y las características generales de un artículo de investigación, tanto respecto del contenido como de la corrección formal y el uso de lenguajes especializados. También se detalla qué habilidades debe poner en funcionamiento para ello el escritor: planificar, revisar de forma crítica las fuentes bibliográficas, justificar la metodología de la investigación y saber analizar tanto cualitativa como cuantitativamente los resultados estadísticos, así como resumir, exponer, argumentar, justificar, etc. Aquí se considera muy importante la presencia del docente de la asignatura para que durante el taller pueda intervenir y esclarecer las dudas que surgen en relación al contexto, al modelo y desarrollo de la investigación. También es positiva su presencia para que los escritores no pierdan de vista al lector final, que es quien recibirá el artículo y lo calificará.

En este taller presencial se presentan las dos principales fases de la elaboración de un artículo científico: la fase de investigación y la de textualización. De este modo, los estudiantes se hacen más conscientes de la necesidad, por un lado, de la importancia de la elección del tema, la búsqueda y selección de información relacionada y la definición de los objetivos de su texto. Por otro, adquieren una visión global de la escritura como un proceso complejo en el que, tras la fase de investigación y la planificación, es necesario elaborar diferentes borradores que se revisan y se mejoran durante el mismo. Durante la segunda parte de esta primera sesión, varios tutores del CEN ayudan a los diferentes grupos de estudiantes en lo relacionado a la superestructura del texto y elaboran el índice de sus artículos. Es importante que siempre quede claro que todo lo relativo al contenido del texto depende de los propios estudiantes dado que son ellos los investigadores.

Entre la primera sesión de intervención formativa del CEN en el aula y la segunda transcurre un mes, tiempo durante el cual los estudiantes disponen de una semana para realizar el Taller de Escritura Académica de Supervivencia (TEAS), un curso en línea elaborado por el CEN al que todos los estudiantes tienen acceso desde su Campus Virtual. Este taller, organizado en seis bloques, combina videos explicativos de carácter divulgativo, fichas de contenido con actividades de autoevaluación y permite a los estudiantes tomar conciencia de la importancia de la redacción textual en el ámbito 
académico, desarrollar las habilidades necesarias para la escritura de textos académicos y profesionales, así como recordar aspectos esenciales de la escritura que les servirán de ayuda para escribir textos precisos, claros, adecuados y correctos (Centro de Escritura Nebrija, 2018).

Para ello, los contenidos del TEAS son los siguientes:

- Bloque 1. Decálogo para la redacción de textos académicos.

a) Presentación del taller online.

b) Decálogo fundamental de supervivencia para redactar textos académicos adecuados.

- Bloque 2. Escribir textos académicos.

a) La planificación.

b) La organización del texto.

c) Tipos de documentos y su estructura.

- Bloque 3. Tipología textual.

a) La exposición.

b) La argumentación.

c) La descripción y la narración.

- Bloque 4. El uso del lenguaje.

a) Puntuación.

b) Ortografía y acentuación.

c) Sintaxis.

d) Léxico.

- Bloque 5. Citación bibliográfica. Plagio.

a) Fuentes.

b) Sistemas de citación.

- Bloque 6. La revisión.

Durante las siguientes tres semanas, los estudiantes, fuera del aula, en grupos, trabajan en la elaboración de sus textos, redactando y revisando diferentes versiones.

Un mes después, en la segunda sesión de intervención del CEN en el aula, los tutores de escritura revisan con los distintos grupos (un tutor por grupo) las versiones más recientes de sus textos, utilizando para ello una rúbrica de evaluación (ver tabla 1). Se lleva a cabo, por tanto, una tutoría entre 
pares grupal de 90 minutos, que ayuda a los escritores a elaborar versiones mejoradas de su primer borrador.

Tabla 1.

Resumen de la rúbrica de evaluación/ revisión ${ }^{1}$

\begin{tabular}{|c|c|c|c|c|}
\hline & & Sí & Dudoso & No \\
\hline $\begin{array}{c}\text { Valoración del } \\
\text { título }\end{array}$ & $\begin{array}{l}\text { 1. Indica claramente el contenido del estudio } \\
\text { 2. Es claro } \\
\text { 3. Es conciso (15 palabras) } \\
\text { 4. Usa tono afirmativo } \\
\text { 5. Usa términos claros y sin siglas } \\
\text { 6. Usa palabras esenciales (etc.) }\end{array}$ & & & \\
\hline $\begin{array}{l}\text { Pautas para } \\
\text { evaluar la } \\
\text { introducción }\end{array}$ & $\begin{array}{l}\text { 1. Se expresa el qué y el porqué de la investigación } \\
\text { 2. El tema general (campo de estudio) se presenta } \\
\text { prontamente para pasar luego al problema de } \\
\text { investigación }\end{array}$ & & & \\
\hline $\begin{array}{l}\text { Problema de } \\
\text { investigación }\end{array}$ & $\begin{array}{l}\text { 1. Se especifica, identifica el problema de } \\
\text { investigación } \\
\text { 2. Se detallan los antecedentes del estudio } \\
\text { 3. Se justifica la elección del tema. } \\
\text { 4. La investigación es viable } \\
\end{array}$ & & & \\
\hline $\begin{array}{c}\text { Revisión } \\
\text { bibliográfica }\end{array}$ & $\begin{array}{l}\text { 1. La revisión bibliográfica es pertinente para el } \\
\text { estudio } \\
\text { 2. La revisión muestra información sobre } \\
\text { antecedentes del problema y permite justificar el } \\
\text { estudio } \\
\text { 3. Las referencias son actuales } \\
\text { 4. (etc.) }\end{array}$ & & & \\
\hline
\end{tabular}

Fuente: elaboración propia.

A continuación, y durante las dos semanas siguientes, los escritores incluyen las sugerencias y mejoras pautadas en la rúbrica y hacen un trabajo de reescritura y revisión final antes de que tenga lugar la tercera sesión en el aula. Es en esta última sesión de intervención del CEN cuando los alumnos entregan su artículo a otro grupo que, con la ayuda del tutor, lo revisa empleando la rúbrica con la que añaden una serie de sugerencias cualitativas al documento. Este se devuelve a sus autores con los comentarios pertinentes para que mejoren y pulan el texto final, que se entrega al profesor de la asignatura.

Puesto que resulta fundamental conocer la opinión de los estudiantes respecto de la experiencia formativa en colaboración con el CEN, al término de la tercera sesión, los estudiantes completan un cuestionario de percepción (ver figura 1) acerca de su experiencia de escritura colaborativa y la intervención formativa. ${ }^{1}$ Solo se incluyen algunos de los aspectos más relevantes de la rúbrica de revisión empleada debido a su extensión y a que
el análisis y la pertinencia del uso de rúbricas no es la finalidad de este artículo. 
Figura 1.

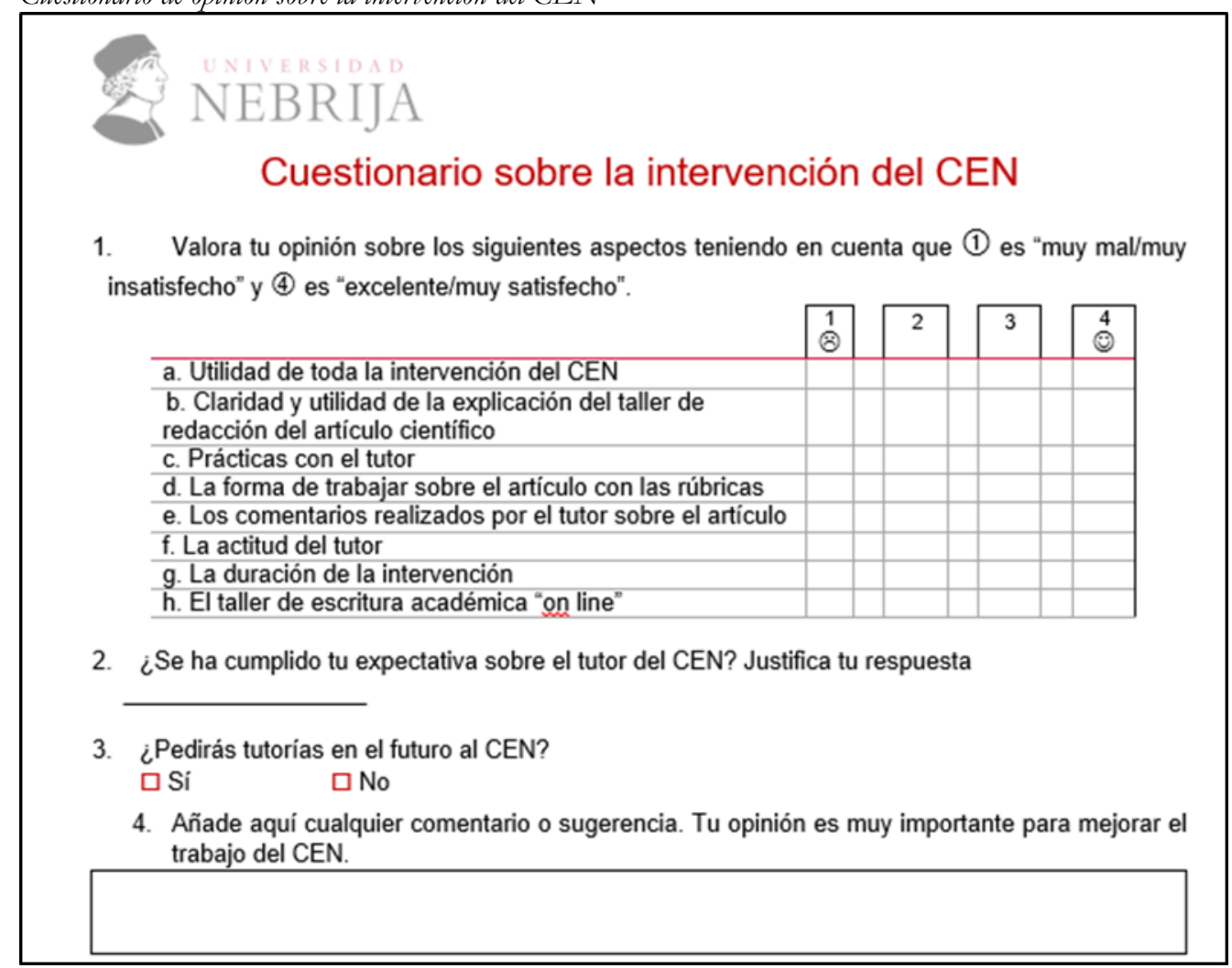

Fuente: elaboración propia.

\section{Resultados}

A continuación, se presenta un resumen de la opinión de los estudiantes y el docente involucrados en la experiencia descrita. Por un lado, mediante un cuestionario de opinión (ver figura 1) que se administra al finalizar la última sesión de la intervención formativa se recopila información sobre la percepción de los estudiantes acerca del TEAS y diferentes aspectos de la actividad colaborativa. Por otro lado, resulta de interés conocer la opinión del docente, por lo que se le realiza una entrevista sobre el impacto de la experiencia en la calidad de los textos y en la adquisición de habilidades investigadoras de los estudiantes un mes después de finalizar la intervención.

Respecto de la percepción de los estudiantes sobre el TEAS; en primer lugar, cabe señalar que el $76 \%$ de los participantes lo realizan, total o parcialmente, en el tiempo establecido, lo que demuestra el alto grado de interés que esta intervención suscita. Conviene mencionar que el $24 \%$ restante corresponde a la mayoría de los estudiantes internacionales (13 de 15) matriculados en la asignatura Diseños de investigación en Psicología quienes, debido a una serie de problemas técnicos, no pudieron acceder a este taller formativo, por lo que su opinión no queda reflejada en este estudio. En segundo lugar, llama la atención que la totalidad de los alumnos que realizan el TEAS completan el bloque IV (El uso del lenguaje) mientras que algunos de ellos dejan ciertas actividades de otros bloques sin completar. En cuanto al nivel de consecución de este taller, el $67 \%$ de los estudiantes que lo realizaron obtienen calificaciones finales de entre 7 y 9 , lo que muestra un alto nivel de aprovechamiento de los contenidos. Sin embargo, aunque el $65 \%$ de los estudiantes valora el TEAS como «muy útil» e «interesante», la puntuación media que le otorgan a este taller en línea en el cuestionario de valoración final evidencia un grado de satisfacción medio (puntuación media de 6) (ver Figura 2). 
Figura 2.

Resumen de los resultados de la percepción de los estudiantes ${ }^{2}$

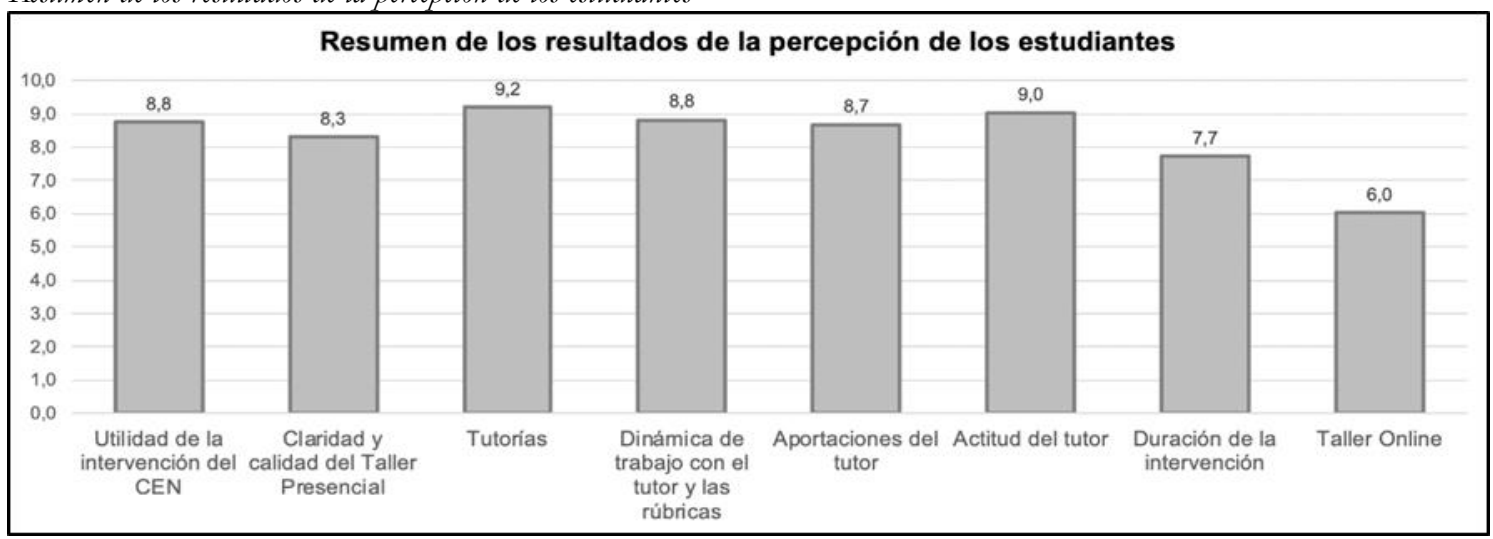

Fuente: elaboración propia.

En cuanto a la opinión global de los estudiantes sobre la intervención formativa del CEN en el aula (ver Figura 2), se puede observar que la consideran de gran utilidad para el desarrollo de su competencia escrita (puntuación media de 8,8), valorando como muy positivas la claridad del taller presencial sobre artículos científicos (puntuación media de 8,3), las tutorías grupales y la dinámica de trabajo propuesta con los tutores (puntuaciones medias de 9,2 y 8,8, respectivamente), la actitud de estos (puntuación media de 9) y sus aportaciones durante la fase de escritura y revisión de los textos (puntuación media de 8,7) (ver figura 2). Asimismo, la duración de la intervención, que se extiende a lo largo de un mes y medio, les parece adecuada (puntuación media de 7,7). En general, se observa un alto grado de satisfacción con la intervención del CEN en el aula.

Es importante señalar que haber generado un ambiente favorable para la escritura colaborativa entre el docente de la asignatura, sus alumnos y los tutores del CEN favorece la construcción de textos académicos adecuados en el contexto universitario. Es más, debido a la relación establecida durante la primera de las sesiones de aula entre los tutores y los escritores, varios de los participantes piden tutorías al CEN en más de una ocasión para trabajar en distintas fases del texto durante el mes de trabajo autónomo en grupos. En estas tutorías se tratan cuestiones como la planificación de ideas en la fase de pre-escritura y se realizan diferentes revisiones y ediciones de los artículos. Además, a lo largo del semestre, tres de los participantes de esta intervención volvieron a acudir al CEN para recibir ayuda con trabajos escritos de otras asignaturas.

Por su parte, el docente reconoce en la entrevista que los autores mejoran la calidad de sus textos a medida que avanzan en la elaboración de los diferentes borradores y que la calidad de los productos escritos, tanto en forma como en contenido, es muy superior a lo esperado en estudiantes de segundo curso de grado. Asimismo, confirma que los artículos presentados reflejan una adecuada organización textual, que responden a los objetivos que se plantean sus autores y que presentan una progresión temática correcta. Por último, señala que la gramática y la ortografía de la mayoría de los artículos le han parecido correctas. En cuanto al desarrollo de las habilidades investigadoras de los estudiantes, el docente afirma que la mayoría de ellos realizan una precisa revisión de las fuentes consultadas, lo que les permite esbozar un adecuado marco teórico. Si bien comenta que, en algunos casos, los escritores no dominan completamente el uso de las fuentes bibliográficas. En resumen, el docente de la asignatura muestra su satisfacción al señalar que la nota media final de la asignatura se ve incrementada gracias a los favorables resultados en los trabajos producidos como resultado de esta intervención formativa.

Lamentablemente, al ser la primera vez que se lleva a cabo una experiencia de estas características en la Universidad Nebrija, no se tienen datos para establecer comparaciones con estudios previos. No obstante, conviene destacar que, de los 12 artículos que se escriben durante el desarrollo de esta

2 Dado que el cuestionario hace uso de una escala de Likert de cuatro puntos, se ha realizado la trasposición de los resultados a una escala 0-10 para facilitar su interpretación. 
experiencia, uno de ellos llega a publicarse en una revista deportiva de carácter divulgativo, lo que no solo aumenta la autoestima y la motivación de los escritores, sino que sirve de incentivo para futuras experiencias.

\section{Conclusiones}

En este artículo se recoge la experiencia de alfabetización académica llevada a cabo en la asignatura de Diseños de investigación en Psicología y realizada por el Centro de Escritura Nebrija. La intervención tiene como objetivos potenciar que los escritores transmitan sus conocimientos en técnicas de investigación y estadística y los documenten e informen en un artículo científico. La experiencia se desarrolla en tres sesiones de aula, en las que participan los estudiantes, el profesor de la asignatura y ocho tutores del equipo del CEN. Las actividades que la conforman, (1) un taller de Redacción de Artículos Académicos, (2) la intervención colaborativa de los tutores en la elaboración de los textos y (3) el Taller online de Escritura Académica de Supervivencia (completado por el $76 \%$ de los participantes), permiten a los estudiantes interactuar con contenido relacionado con la escritura de textos académicos y profesionales, así como beneficiarse de la guía de tutores con mayor nivel de alfabetización académica.

$\mathrm{Al}$ amparo de los resultados analizados, se comprueba que los escritores universitarios valoran positivamente la formación específica de escritura académica, así como el trabajo de colaboración. Una vez que comprenden que la escritura es un proceso, y gracias a un exhaustivo trabajo de revisión de los textos facilitado por los tutores del CEN, los escritores verifican que sus artículos mejoran significativamente y el docente reconoce un incremento cualitativo en los trabajos finales que revisa.

Los resultados muestran que acciones como estas en las que se contemplan los talleres de escritura formativos, las tutorías entre pares y el uso de rúbricas de evaluación para la revisión colaborativa de los textos escritos en el aula tienen un impacto muy positivo, tanto en los resultados de los trabajos realizados, como en los procesos formativos de escritura de los estudiantes universitarios. Además, se fomentan los nuevos entornos de enseñanza y aprendizaje colaborativos, tan apropiados en el desarrollo de las competencias de los futuros profesionales. Con iniciativas como esta se ponen de manifiesto los beneficios de la integración de la escritura en los planes académicos de enseñanza superior.

Se espera poder replicar este modelo de intervención formativa en futuras ediciones, incorporando las mejoras que han surgido a raíz de esta experiencia (cambios en el TEAS, por ejemplo) de manera más controlada, de forma que se puedan analizar las mejoras cualitativas de los textos respecto del primer borrador y del último, permitiendo hacer un estudio cuantitativo, así como poder contrastar los resultados de los artículos de los distintos grupos y en distintos cursos lectivos.

\section{Referencias}

Álvarez Álvarez, M. D. L. N., Villardón Gallego, L., \& Yániz Álvarez de Eulate, C. (2010). Influencias de factores sociocognitivos en la calidad de la escritura en los estudiantes universitarios. Educatio Siglo XXI, 28(2), pp. 181-204. Recuperado de https://revistas.um.es/educatio/article/view/112021

Arrieta de Meza, B., Batista Ojeda, J. T., Meza Cepeda, R. D., \& Meza Corona, D. Y. (2006). La comprensión lectora y la redacción académica como centro del currículum. Laurus, 12(21), pp. 8694. Recuperado de https://dialnet.unirioja.es/servlet/articulo?codigo $=2968913$

Avilán Díaz, A. (2004). La escritura: abordaje cognitivo. (Hacia la construcción de una didáctica cognitiva de la escritura). Acción pedagógica, 13(1), pp. 18-30. Recuperado de https://dialnet.unirioja.es $/$ servlet $/$ articulo?codigo $=2971868$

Britton, J. (1970). Language and learning. Miami, Florida: University of Miami Press.

Caldera, R., \& Bermúdez, A. (2007). Alfabetización académica: comprensión y producción de textos. Educere, 11(37), pp. 247-255. Recuperado de https://www.redalyc.org/pdf/356/35603710.pdf

Camps, A. (2011). Escribir para aprender: una visión desde la teoría de la actividad. Comunicación en XII Congreso de la SEDLL. Recuperado de https://www.researchgate.net/publication/242256893

Carlino, P. (2003). Alfabetización académica: Un cambio necesario, algunas alternativas posibles. Educere, 6(20), pp. 409-420. 
Carlino, P. (2005). Escribir, leer y aprender en la Universidad. Una Introducción a la alfabetización académica. Buenos Aires: Fondo de Cultura Económica. Recuperado de https://www.aacademica.org/paula.carlino/3.pdf

Carlino, P. (2013). Alfabetización académica diez años después. Revista mexicana de investigación educativa, 18(57), pp. 355-381. Recuperado de http://www.scielo.org.mx/scielo.php?script=sci_arttext\&pid=S1405$66662013000200003 \& \operatorname{lng}=\mathrm{es} \& \operatorname{tlng}=\mathrm{en}$

Cartolari, M., \& Carlino, P. (2012). Leer y escribir en la formación docente: aportes de las investigaciones anglosajonas. Acción Pedagógica, 21(1), pp. 6-17. Recuperado de https://www.aacademica.org/paula.carlino/36

Cassany, D., \& Morales, O. A. (2008). Leer y escribir en la universidad: Hacia la lectura y la escritura crítica de géneros científicos. Revista Memoralia, 5, pp. 69-82. Recuperado de https://www.studocu.com/es/u/4118453

Castelló, M., Bañales, G., \& Vega, N. (2010). Enfoques en la investigación de la regulación de escritura académica: Estado de la cuestión. Electronic Journal of Research in Educational Psychology, 8(3), pp. 12531282. Recuperado de https://www.redalyc.org/pdf/2931/293122000015.pdf

Centro de Escritura Nebrija (2018). Taller de Escritura de Supervivencia (TEAS). Centro de Escritura Nebrija, Universidad Nebrija, Madrid, España.

De Beaugrande, R. A., \& Dressler. W. U. (1997). Introducción a la lingüistica. Barcelona: Ariel.

De la Barrera, S., \& Bono, A. (2004). Escribir para aprender mejor en la universidad. Prácticas de escritura en contextos pedagógicos. Acción pedagógica, 13(1), pp. 32-36. Recuperado de https://dialnet.unirioja.es/servlet/articulo?codigo $=2971887$

De la Peña, A. (2019). La escritura colaborativa en la universidad: un estudio de casos en español como lengua extranjera. (Tesis doctoral inédita).

Flower, L., \& Hayes, J. R. (1980). Identifying the Organization of Writing Process. En L. W. Gregg y E. R. Steinberg (Eds.), Cognitive processes in writing: An Interdisciplinary Approach, pp. 3-30. Hillsdale, N. J.: Lawrence Erlbaum. Recuperado de https://www.researchgate.net/publication/200772468_Identifying_the_organization_of_writing_processes

Gallego Ortega, J. L., \& Mendías Cuadros, A. M. (2012). ¿Qué saben los estudiantes universitarios sobre la planificación de tareas de escritura? Un estudio de caso. Revista de investigación en educación, 10(2), pp. 47. Recuperado de https://dialnet.unirioja.es/servlet/articulo?codigo $=4732994$

García Negroni, M. M. \& Hall, B. (2010). Escritura universitaria, fragmentariedad y distorsiones enunciativas propuestas de prácticas de lectura y escritura focalizadas en la materialidad lingüísticodiscursiva. Boletín de lingüistica, XXII (34), pp. 41-69. Recuperado de https://www.redalyc.org/articulo.oa?id $=34721061003$

Gibbs, G., \& Simpson, C. (2004). Conditions under which assessment supports learning. Learning and Teaching in Higher Education, 1, pp. 3-31. Recuperado de http://eprints.glos.ac.uk/3609/1/LATHE\%201.\%20Conditions\%20Under\%20Which\%20Assessment $\% 20$ Supports $\% 20$ Students $\% 27 \% 20$ Learning\%20Gibbs_Simpson.pdf

Gutiérrez-Rodríguez, M. J., \& Flórez-Romero, R. (2011). Enseñar a escribir en la universidad: saberes y prácticas de docentes y estudiantes universitarios. Magis, Revista Internacional de Investigación en Educación, 4(7), pp. 137-168. Recuperado de https://revistas.javeriana.edu.co/index.php/MAGIS/article/view/3561

Herring, S. (Ed). (1984). Computer-Mediated Communication. Linguistic, social and cross-cultural perspectives. Amsterdam, Filadelfia: John Benjamins Publishing Company. doi: 10.1075/pbns.39

Ibarra, M. S., \& Rodríguez, G. (2010). Los procedimientos de evaluación como elementos de desarrollo de la función orientadora en la universidad. Revista Española de Orientación y Psicopedagogía, 21(2), pp. 443-461. doi: 10.5944/reop.vol.21.num.2.2010.11558

Kern, R. (2006). Perspectives on technology in learning and teaching languages. TESOL Quarterly, 40(1), pp. 183-210. doi: 10.2307/40264516

Lacon, N. \& Ortega, S. (2008). Cognición, metacognición y escritura. Revista Signos, 41, pp. 231-255. doi: 10.4067/S0718-09342008000200009

Lavellee, E. (2003). The Quality of University writing: a preliminary analysis of undergraduate portfolios. Quality on Higher Education, 9(1), pp. 87-93. doi: 10.1080/13538320308159

Lea, M. R. \& Street, B. V. (1998). Student writing in higher education: an academic literacies approach. Studies in Higher Education, 23(2), pp. 157-172. doi: 10.1080/03075079812331380364 
Marín, M. (2006). Alfabetización académica temprana. Lectura y vida. Revista Latinoamericana de Lectura, 27(4), pp. 30-39. Recuperado el 13 de abril de 2020, de https://media.utp.edu.co/referenciasbibliograficas/uploads/referencias/articulo/751-alfabetizacion-academica-tempranapdf-sSJKgarticulo.pdf

Marín, J., López, S., \& Roca-De-Larios, J. (2015). El proceso de escritura académica en la universidad española: percepciones de estudiantes y profesores. Educación y Cultura, 27(3), pp. 504-533. doi: 10.1080/11356405.2015.1072360

Marinkovich, J., Morán, P., \& Vergara, M. (1996). Hacia una representación del proceso de producción escrita en el aula. Revista Signos, 29(40), pp. 107- 115.

Milian, M. (2001). Interacción de contextos en la investigación sobre la composición escrita. En Camps, A. (Coord.), El aula como espacio de investigación y reflexión (pp. 23-36). Barcelona: Grao.

Núñez Cortés, J. A. (2013). La alfabetización académica: estudio comparado en el ámbito Iberoamericano. (Tesis doctoral, Universidad Autónoma de Madrid). Recuperado de http://hdl.handle.net/10486/13256

Ochoa, S., \& Aragón, L. (2007). Funcionamiento metacognitivo de estudiantes universitarios durante la escritura de reseñas analíticas. Universitas Psychologica, 6(3), pp. 493-506. Recuperado de http://pepsic.bvsalud.org/scielo.php?script=sci_arttext\&pid=S1657$92672007000300003 \& \operatorname{lng}=\mathrm{es} \& \operatorname{lng}=\mathrm{es}$

Puerta, C. A., \& Sánchez, L. M. (2010). Escritura y lectura en ambientes virtuales de enseñanzaaprendizaje como proceso formativo. En A. A. Sánchez Upegui, C. A. Puerta Gil \& L. M. Sánchez Ceballos (2010). Manual de comunicación en ambientes educativos virtuales (pp. 37- 82). Medellín, Colombia: Fundación Universitaria Católica del Norte. Recuperado de https://www.ucn.edu.co/institucion/sala-prensa/Documents/Libro_Educacion_Virtual-_Julio_01_de_2010-_Version_Final.pdf

Reguera, A. (2010). Características de la argumentación en textos académicos producidos por estudiantes universitarios. En V. M. Castel, \& L. Cubo (Eds.), La renovación de la palabra en el bicentenario de la Argentina. Los colores de la mirada lingüística (pp. 1109-1115). Mendoza: Editorial FfyL. Recuperado de https://pdfs.semanticscholar.org/4f72/2dd978dcad66a5e08992e827b7e345e0c71b.pdf?_ga=2.204636688.106971829.1586 $774412-1480542060.1561890589$

Rojas, G., \& Jiménez, H. (2012). La educación superior desde la lectura y la escritura. Amazonia Investiga, 3(1), pp. 19-35. Recuperado de https://amazoniainvestiga.info/index.php/amazonia/article/view/657

Sabaj, O. (2009). Descubriendo algunos problemas en la redacción de Artículos de Investigación Científica (AIC) de alumnos de postgrado. Revista Signos, 42(69), pp. 107- 127. doi: 10.4067/S071809342009000100006

Tapia, M., Burdiles, G., \& Arancibia, B. (2003). Aplicación de una pauta diseñada para evaluar informes académicos universitarios. Revista Signos, 36(54), pp. 249-257. doi: 10.4067/S071809342003005400009

Vázquez, A. (2005). ¿Alfabetización en la universidad? En Secretaria Académica, Colección de cuadernillos de actualización para pensar la enseñanza universitaria, 1(1), pp. 4-7. Recuperado de http://www.eemn1 tsas.edu.ar/Autoevaluacion/Vasquez-Alfabetizacion $\% 20 \mathrm{en} \% 201 \mathrm{a} \% 20$ Universidad.pdf

Vigotsky, L. S. (1978). Mind in society: The development of higher psychological processes. Cambridge, MA: Harvard University Press.

Wertsch, J. (1991). Voices of the mind. A Sociocultural Approach to Mediated Action. Cambridge, MA: Harvard University Press. 